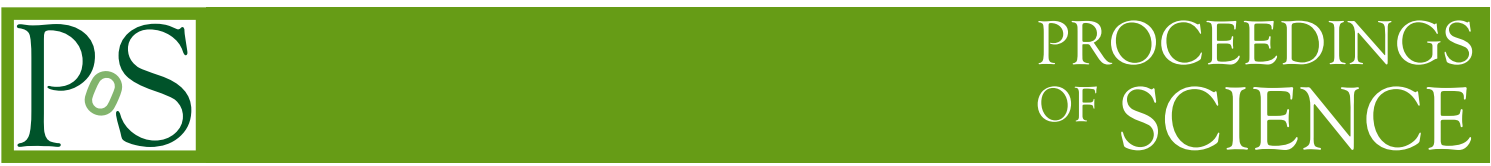

\title{
FRUITY upgrades on AGB nucleosynthesis
}

\author{
Sergio Cristallo* \\ INAF - Osservatorio Astronomico di Teramo, Italy \\ INFN - Sezione di Napoli, Italy \\ E-mail: sergio.cristallo@inaf.it
}

\section{Oscar Straniero}

INAF - Osservatorio Astronomico di Teramo, Italy

INFN - Sezione di Napoli, Italy

E-mail: oscar.straniero@inaf.it

\section{Luciano Piersanti}

INAF - Osservatorio Astronomico di Teramo, Italy

INFN - Sezione di Napoli, Italy

E-mail: luciano.piersanti@inaf.it

Asymptotic Giant Branch (AGB) stars efficiently pollute the interstellar medium, producing both light ( $\mathrm{C}, \mathrm{N}, \mathrm{O}, \mathrm{F}, \mathrm{Na})$ and heavy elements (via the slow neutron capture process, the s-process). The major neutron source is the ${ }^{13} \mathrm{C}(\alpha, \mathrm{n}){ }^{16} \mathrm{O}$ reaction, mainly working in low-mass AGB stars $\left(\mathrm{LM}-\mathrm{AGBs}, 1.2<\mathrm{M} / \mathrm{M}_{\odot} \leq 4.0\right)$ and responsible for the production of the main component of the sprocess. An additional contribution comes from the ${ }^{22} \mathrm{Ne}(\alpha, \mathrm{n})^{25} \mathrm{Mg}$ reaction, which is efficiently activated in intermediate-mass AGB stars (IM-AGBs, $4.0<\mathrm{M} / \mathrm{M}_{\odot} \leq 6.0$ ). Our AGB models are available on the web pages of the FRUITY database. New sets of AGB models are presented here: a first one including a full range of IM-AGBs at different metallicities (4.0, 5.0 and 6.0 $\mathrm{M}_{\odot}$ with $-2.15 \leq[\mathrm{Fe} / \mathrm{H}] \leq+0.15$ ) and a second one of LM-AGBs including rotation. We show how the two neutron sources differently contribute, as a function of the initial mass, to the surface patterns. Then, we discuss the effects of rotation on the LM-AGBs nucleosynthesis. In these objects the dominant neutron source mostly burns in radiative conditions; thus, the presence of secular rotation induced mixing during the interpulse phase leads to appreciable changes in the final s-process surface distributions. We present comparisons with available spectroscopic data at different metallicities and laboratory isotopic measurements of pre-solar SiC grain.

XIII Nuclei in the Cosmos

7-11 July, 2014

Debrecen, Hungary

\footnotetext{
${ }^{*}$ Speaker.
} 


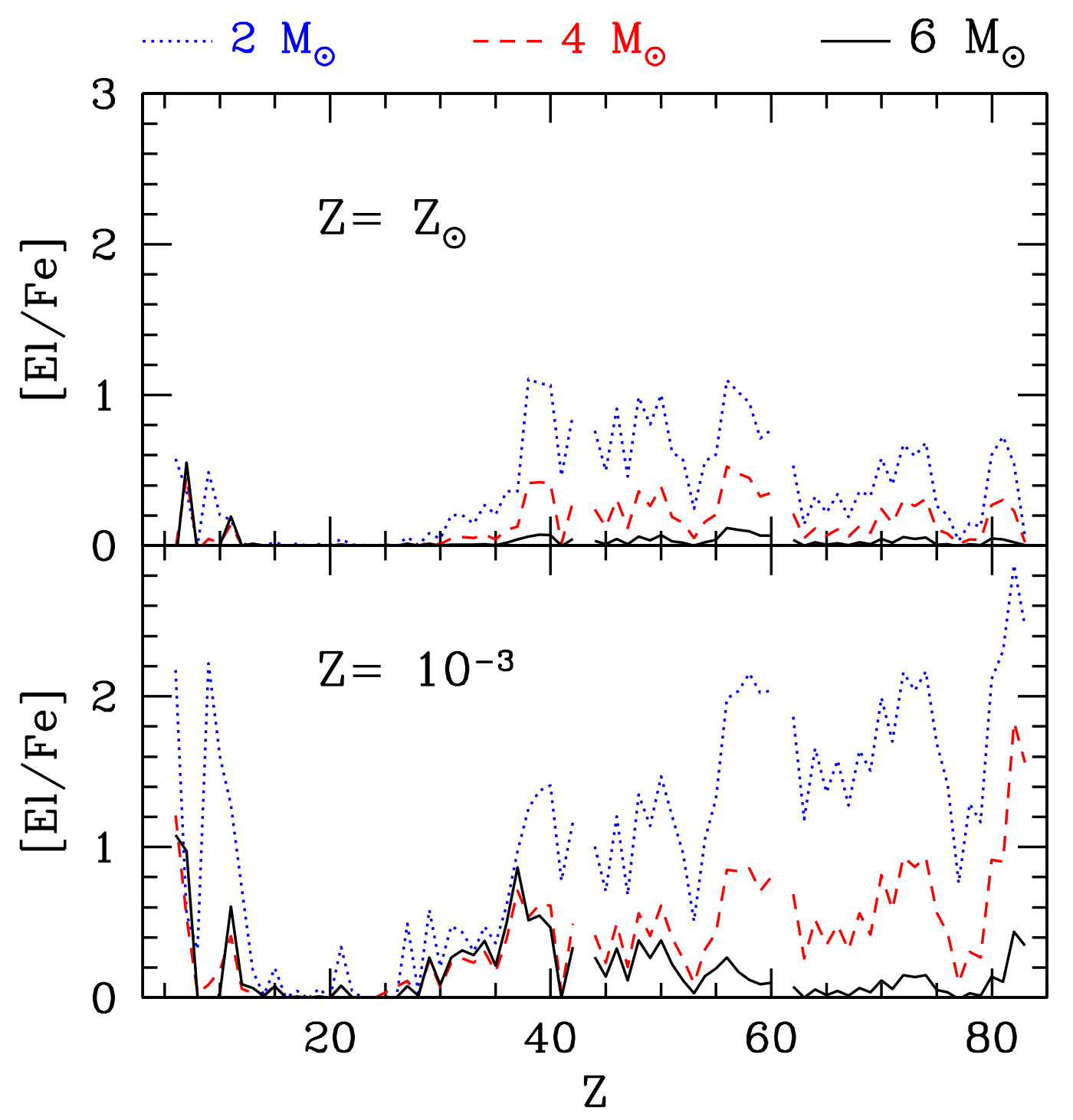

Figure 1: Final surface s-process distributions in AGB models with different masses and metallicities.

\section{FUNS AGB models and the FRUITY database}

Isotopes heavier than iron $(\mathrm{A} \geq 56)$ are synthesized by neutron capture processes only (apart from a restricted number of proton-rich nuclei). Observed spectroscopic heavy element distribution show the existence of two main components, correlated to different nucleosynthetic processes: the $\mathrm{s}$ (slow) process and the $\mathrm{r}$ (rapid) process. The $\mathrm{r}$ process requires high neutron densities, and it is believed to occur during explosive phases of stellar evolution (neutron stars mergers). The s process, characterized by a slow neutron capture with respect to the corresponding $\beta$ decay, is at work in AGB stars [1]. During this evolutionary phase, elements synthesized in the internal layers are carried out to the surface via recurrent mixing episodes called Third Dredge Ups, TDUs (for reviews on AGB stars and the s-process see [2], [3] and [4]). Main neutron sources are the ${ }^{13} \mathrm{C}(\alpha, \mathrm{n}){ }^{16} \mathrm{O}$ 
reaction, active in radiative layers during the interpulse period [5], and the ${ }^{22} \mathrm{Ne}(\alpha, \mathrm{n})^{25} \mathrm{Mg}$ reaction, activated at the base of convective shells driven by Thermal Pulses (TPs). In order to obtain a sufficient amount of ${ }^{13} \mathrm{C}$ for a consistent s-process production, a diffusion of protons below the inner border of the convective envelope is requested during a TDU episode. Then, ${ }^{13} \mathrm{C}$ is synthesized via the ${ }^{12} \mathrm{C}(\mathrm{p}, \gamma){ }^{13} \mathrm{~N}\left(\beta^{-}\right){ }^{13} \mathrm{C}$ nuclear chain, leading to the formation of the so-called ${ }^{13} \mathrm{C}$-pocket. While the ${ }^{13} \mathrm{C}(\alpha, \mathrm{n}){ }^{16} \mathrm{O}$ reaction provides the bulk of the s-process, the ${ }^{22} \mathrm{Ne}(\alpha, \mathrm{n}){ }^{25} \mathrm{Mg}$ reaction marginally contributes to the final s-process AGB surface distributions. However, if the temperature attained during a TP exceeds $300 \mathrm{MK}$, this reaction provide a non negligible contribution to the first peak of the s-process and to the production of neutron rich isotopes.

Our AGB models have been computed with the FUNS code ([4] and references therein). Recent upgrades to the code as well as a detailed description of the network adopted to run the calculations can be found in [6] and [7]. The chemical features of our models are available on the web pages of the FRUITY database, which contains AGB isotopic, elemental and yields tables [8]. The stellar evolution is followed from the pre Main Sequence up to the tip of the AGB, when the tiny residual envelope mass does not allow TDU to occur. FRUITY is currently hosting more than 50 models of LM-AGB stars $\left(1.3 \leq \mathrm{M} / \mathrm{M}_{\odot} \leq 3.0\right)$ with metallicities ranging from $[\mathrm{Fe} / \mathrm{H}]=-2.15$ to $[\mathrm{Fe} / \mathrm{H}]=+0.15$.

\section{Intermediate Mass AGB stars}

We expanded our database by computing a new set of IM-AGBs $\left(4.0,5.0\right.$ and $\left.6.0 \mathrm{M}_{\odot}\right)$ at all FRUITY metallicities. In Figure 1 we show a comparison between models with different masses (2.0, 4.0 and $\left.6.0 \mathrm{M}_{\odot}\right)$ and metallicities $\left(Z=10^{-3}\right.$ and $\left.Z=Z_{\odot}\right)$. As expected, in low mass stars the lower the metallicity the larger the contribution to the second and third peak of the s-process (the Ba-La-Nd region and ${ }^{208} \mathrm{~Pb}$, respectively). This is due to the fact that while iron seeds scale with the metallicity, the neutron source (i.e. the ${ }^{13} \mathrm{C}$ ) is of primary origin. Thus, the neutron-to-seed ratio increases at low $\mathrm{Z}$ and, therefore, heaviest isotopes can be produced. This explanation cannot be applied to more massive AGBs, as demonstrated in Figure 1. In those objects, in fact, the increased compactness of the structure as well as the larger temperature at the base of the convective envelope during TDUs do not allow the formation of large ${ }^{13} \mathrm{C}$ pockets (as in LM-AGBs). As a result, the dominant contribution to s-process nucleosynthesis comes from the ${ }^{22} \mathrm{Ne}(\alpha, \mathrm{n})^{25} \mathrm{Mg}$ reaction, which is responsible for a consistent production of elements belonging to the first s-process peak ( $\mathrm{Sr}-\mathrm{Y}$ $\mathrm{Zr}$ ) as well as some neutron rich isotopes (as ${ }^{87} \mathrm{Rb}$ and ${ }^{96} \mathrm{Zr}$ ). In general, however, the reduced TDU efficiency characterizing these objects [11] does not allow large envelope enrichments and, as a consequence, the contribution from IM-AGBs to the galactic chemical evolution is almost negligible [10].

\section{Rotating AGB models}

As shown in [8], FRUITY models cannot reproduce the spread in s-process distributions observed at different metallicities. In particular, a mechanism able to reduce the neutron-to-seed ratio is requested. We showed that rotation can help to reconcile models to observations [10]. In rotating AGB stars, in fact, additional non-convective mixing can be triggered by meridional circulations or Goldreich-Schubert-Fricke instabilities. In particular, the latter mixes downward the abundant ${ }^{14} \mathrm{~N}$ 


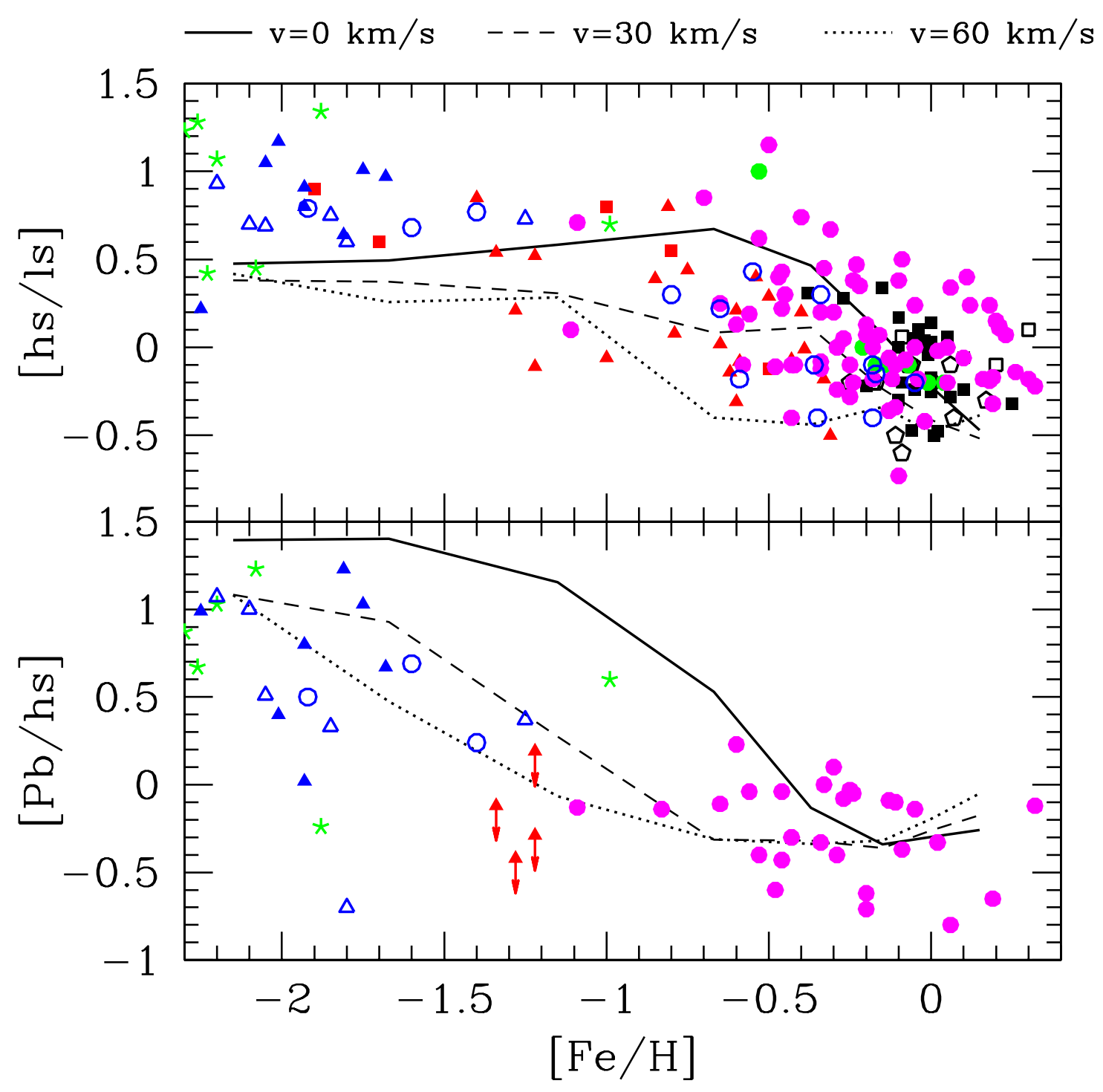

Figure 2: Surface s-process indexes in low mass stars at different metallicities with different initial rotational velocities. Observational data are shown as a comparison. See text for details.

present in the upper layers of the ${ }^{13} \mathrm{C}$ pocket. As a net effect, the neutron-to-seed ratios decreases, as ${ }^{14} \mathrm{~N}$ is the major neutron poison via the ${ }^{14} \mathrm{~N}(\mathrm{n}, \mathrm{p}){ }^{14} \mathrm{C}$ reaction. The larger the initial rotational velocity, the larger the effects induced by rotation on the final s-process distributions. In Figure 2 we report s-process indexes ${ }^{1}$ as measured in a wide variety of objects (O-rich intrinsic AGB stars; C-rich intrinsic AGB stars; SC stars; post-AGB stars; CH stars; CEMP-s stars; CEMP-sr stars). Rotating models help to fit [hs/ls] observations at intermediate-to-large metallicities $-1<[\mathrm{Fe} / \mathrm{H}]<0$ and, at the same time, improve the fit to $[\mathrm{Pb} / \mathrm{hs}]$ at low $\mathrm{Z}$. Notwithstanding, there are still data not reproduced by our theoretical models. Thus, a further effort is needed in this direction.

$\left.\left.{ }^{1}[\mathrm{hs} / \mathrm{Fe}]=([\mathrm{Ba} / \mathrm{Fe}]+[\mathrm{La} / \mathrm{Fe}]+[\mathrm{Nd} / \mathrm{Fe}]+[\mathrm{Sm} / \mathrm{Fe}]) / 4\right) ; \quad[1 \mathrm{~s} / \mathrm{Fe}]=([\mathrm{Sr} / \mathrm{Fe}]+[\mathrm{Y} / \mathrm{Fe}]+[\mathrm{Zr} / \mathrm{Fe}]) / 3\right) ; \quad[\mathrm{hs} / \mathrm{ls}]=[\mathrm{hs} / \mathrm{Fe}]-[\mathrm{ls} / \mathrm{Fe}] ;$ $[\mathrm{Pb} / \mathrm{hs}]=[\mathrm{Pb} / \mathrm{Fe}]-[\mathrm{hs} / \mathrm{Fe}]$. The $1 \mathrm{~s}$, the hs and $\mathrm{Pb}$ represent the three s-process peaks. 


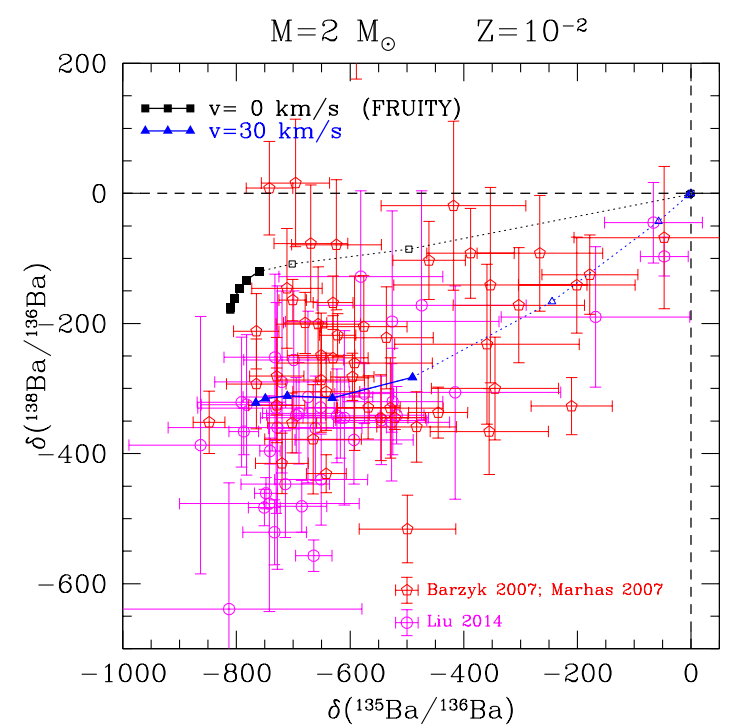

Figure 3: Barium isotopic ratios in a FRUITY $2 \mathrm{M}_{\odot}$ star with $Z=10^{-2}$ compared to isotopic laboratory measurements in pre-solar grains. A rotating model with initial rotational velocity $\mathrm{v}=30 \mathrm{~km} / \mathrm{s}$ is also shown.

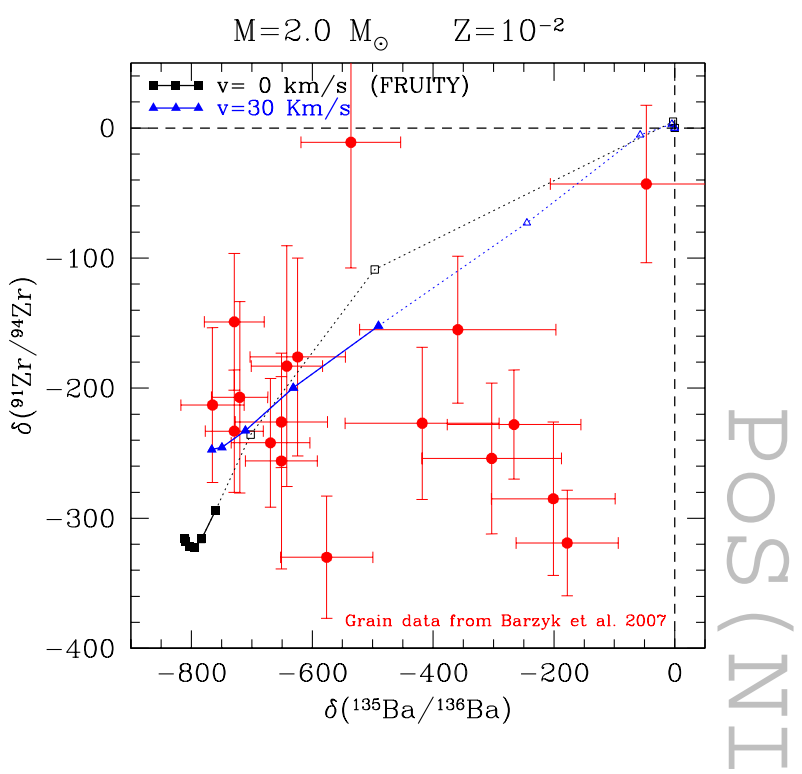

Figure 4: As in Figure 3 but including zirconium isotopic ratios. Zirconium and barium data have been simultaneously extracted from the same grains.

Another way to check the reliability of our theoretical models is to compare them to laboratory isotopic measurement in pre-solar silicon carbide ( $\mathrm{SiC}$ ) grains. Those pristine microcrystals condensed in carbon-rich stellar winds; later, they have been ejected into the interstellar medium preserving their nucleosynthetic origin, transported to the protosolar nebula and finally incorporated in meteorite parent bodies. Considering the small errors affecting their isotopic ratios measurements, pre-solar Sic grains provide the most precise available nucleosynthetic data and, thus, strongly constrain theoretical models (see e.g. [12], [13], [14], [15]). S-process isotopic ratios mainly depend on the neutron-to-seed ratio (and thus on the ${ }^{13} \mathrm{C}$ profile in the pocket), even if they can also vary with the mass and the metallicity. In Figure 3 we show the surface barium isotopic ratios evolution of a $2 \mathrm{M}_{\odot}$ AGB star with $Z=10^{-2}$ and different initial rotational velocities ( 0 and $30 \mathrm{~km} / \mathrm{s}$ ), compared with extant $\mathrm{SiC}$ data ([16], [17], [13]). The inclusion of rotation definitely improves our fit to grains, thus confirming the need of a process able to reduce the neutron-to-seed ratio in our ${ }^{13} \mathrm{C}$ pockets. This is also confirmed by Figure 4, where we report multi-element isotopic ratios derived in the same grains [16]. Those measurements constrain models even more than those reported in Figure 3, because isotopic ratios for elements corresponding to different peaks of the s-process have to be reproduced. Also in this case, a better fit to grains data is found with the rotating AGB model.

\section{References}

[1] Gallino, R., and 7 coauthors.: 1998, ApJ 497, 388.

[2] Busso, M., Gallino, R., Wasserburg, G. J.: 1999, ARA\&A 37, 239.

[3] Herwig, F., 2005, ARA\&A 43, 435 
[4] Straniero, O., Gallino, R., \& Cristallo, S., 2006, Nucl. Phys. A 777, 311

[5] Straniero, O., Gallino, R., Busso, M., Chieffi, A., Limongi, M., \& Salaris, M., 1995, ApJ 440, L85

[6] Cristallo, S., Straniero, O., Lederer, M.T., \& Aringer, B., 2007, ApJ 667, 489

[7] Cristallo, S., Straniero, O., Gallino, R., Piersanti, L., Domínguez, I., \& Lederer, M.T., 2009, ApJ 696, 797

[8] Cristallo, S., Piersanti, L., Straniero, O., Gallino, R., Domínguez, I., Abia, C., Di Rico, G., Quintini, M., \& Bisterzo, S., 2011, ApJS 197, 17

[9] Straniero, O., Cristallo, S., \& Piersanti, L., 2014, ApJ 785, 77

[10] Cristallo, S., Abia, C., Straniero, O., \& Piersanti, L., ApJ submitted

[11] Piersanti, L., Cristallo, S., \& Straniero, O., 2013, ApJ 774, 98

[12] Lugaro, M., Tagliente, G., Karakas, A.I., Milazzo, P.M., Käppeler, F., Davis, A.M., \& Savina, M.R., 2014, ApJ 780, 95

[13] Liu, N., Savina, M.R., Davis, A.M., Gallino, R., Straniero, O., Gyngard, F., and 13 coauthors, 2014, ApJ 786, 66

[14] Liu, N., Gallino, R., Bisterzo, S., Davis, A.M., Savina, M.R., \& Pellin, M.J. 2014, ApJ 788, 163

[15] Liu, N., et al., ApJ submitted

[16] Barzyk, J.G., Savina, M.R., Davis, A.M., Gallino, R., Gyngard, F., and 5 coauthors, 2007, Meteoritics \& Planetary Science, 42, 1103

[17] Marhas, K.K., Hoppe, P., \& Ott, U., 2007, Meteoritics \& Planetary Science, 42, 1077 